\title{
New diagnosis of myasthenia gravis presenting as myasthenic crisis after radical thymectomy: a case report
}

\author{
Rahul Nayak $^{1}$, Ryan Rebello ${ }^{2}$, Yaron Shargall ${ }^{1}$ \\ ${ }^{1}$ Division of Thoracic Surgery, Department of Surgery, ${ }^{2}$ Department of Diagnostic Imaging, McMaster University, Hamilton, ON, Canada \\ Correspondence to: Yaron Shargall, MD. Division of Thoracic Surgery, Department of Surgery, McMaster University, St. Joseph's Healthcare Hamilton \\ Charlton Campus, 50 Charlton Avenue East, Room T2105, Hamilton, ON, L8N 4A6, Canada. Email: Shargal@mcmaster.ca.
}

\begin{abstract}
Myasthenia gravis (MG) is a well-known paraneoplastic syndrome associated with thymomas. It occurs secondary to the production of autoantibodies against acetylcholine receptors (AChR). This results in the reduced delivery of action potentials that generate muscular contractions. In its most extreme form, MG can present as a crisis resulting in respiratory failure requiring invasive ventilatory support. There are several known risk factors for patients with known MG to develop a post-operative crisis after thymectomy. It is recommended that patients diagnosed with thymoma be evaluated for MG prior to resection. However, most guidelines do not make any recommendations regarding screening with AChR antibody tests if there are no signs or symptoms of MG. In this case report, we present a patient with an incidental finding of a large anterior mediastinal thymoma with no signs or symptoms of MG. The patient subsequently develops bulbar symptoms and dyspnea necessitating readmission to hospital 2 weeks after surgery. This eventually culminated in cardiorespiratory arrest. This was confirmed to be secondary to a myasthenic crisis via AChR autoantibody testing. The patient was treated with intravenous immunoglobulin (IVIG), pyridostigmine and prednisone with good effect. The onset of myasthenic crisis after a surgical insult in known MG patients has been well documented. However, the development of myasthenic crisis in an asymptomatic patient with no history of MG has not previously been shown. This case report illustrates the need for more sensitive screening tools to detect MG in patients with thymoma. The routine use of AChR autoantibody testing as a screening tool in patients with thymoma even when asymptomatic and/or deemed low risk may help clinicians better prepare for possible MG flairs. Furthermore, surgeons should have a low threshold to suspect MG in patients after thymectomy regardless of time elapsed since surgery.
\end{abstract}

Keywords: Thymoma; myasthenia gravis (MG); myasthenic crisis; case report; thymectomy

Received: 12 May 2020; Accepted: 10 July 2020; Published: 25 May 2021.

doi: $10.21037 /$ ccts-2020-06

View this article at: http://dx.doi.org/10.21037/ccts-2020-06

\section{Introduction}

Myasthenia gravis (MG) refers to an autoimmune condition resulting in progressive muscle weakness with repetitive use. Specifically, the disorder results from the production of autoantibodies against acetylcholine receptors (AChR) or muscle-specific tyrosine kinase (MuSK) (1). An assay can be performed on patients' blood samples to detect the presence of antibodies against AChR and MuSK. This will return positive in approximately $85 \%$ of cases (2). Binding of these autoantibodies to their target receptor leads to impaired neuromuscular transmission and muscle weakness. Consequently, treatment of MG focuses on two main pathways. The first aims to decrease the production of autoantibodies via immune modulation (i.e., steroids, immunosuppressants). The second approach aims to increase concentrations of acetylcholine at the neuromuscular junction by competing against these autoantibodies (i.e., pyridostigmine). Given the lower adverse effect profile with pyridostigmine, the latter approach is preferred for maintenance therapy. In emergent scenarios, rapid removal of antibodies is required. 
This is performed via plasmapheresis or intravenous immunoglobulin (IVIG) (3).

The incidence of MG is estimated to be 2.0-5.0 per million per year with a female predominance (4). However, thymoma-associated MG does not have a gender predilection and occurs between the ages of 40-60 years (4). The level of dysfunction from MG is categorized via the Osserman classification system. Osserman I indicates purely ocular MG, while Osserman V refers to respiratory failure from MG. Patients with Osserman II-IV display varying degrees of bulbar and extremity weakness (5). A randomized controlled trial of patients with non-thymomatous MG and Osserman II-IV dysfunction showed improvement in patients' symptoms and reduction in medication use with thymectomy compared to medical management after a 3 -year interval (6). Consequently, thoracic surgeons will need to familiarize themselves with this entity as they will increasingly receive referrals for consideration of thymectomy for myasthenia patients.

The diagnosis of MG is typically made prior to thymectomy and resection is intended to help with reduction of symptoms and medication requirements. We present a case of MG in a lifelong asymptomatic patient with no known MG history resulting in life threatening myasthenic crisis approximately 3 weeks after thymectomy. To the best of our knowledge, this is the first case to highlight a delayed and dramatic new diagnosis of MG after a thymectomy. We present the following case in accordance with the CARE reporting checklist (available at https://ccts. amegroups.com/article/view/10.21037/ccts-2020-06/rc).

\section{Case presentation}

A 46-year-old male presented with an incidental finding of an anterior mediastinal mass (MM) on a chest X-ray during routine pre-operative evaluation for a nasal septoplasty. $\mathrm{He}$ was a non-smoker with no significant medical or surgical history. He did not demonstrate any signs or symptoms concerning for superior vena cava (SVC) syndrome or airway collapse with position changes. He did not endorse any weight loss, lethargy, fevers, chills or night sweats. $\mathrm{He}$ did not endorse any ocular, bulbar or extremity muscle weakness on history suggestive of MG. He did not have any family history of $M G$ or any neuromuscular disorders. His physical exam did not reveal any ptosis or fatiguability with repetitive motions. Given his lack of symptoms and lack of any prior history raising suspicion for MG he was not sent for any further testing with regards to MG prior to surgery. The MM was further investigated with computer tomography (CT) scan of the chest, a tumor marker panel for lactate dehydrogenase (LDH), alphafetoprotein (AFP) and beta-human chorionic gonadotropin (B-HCG) and a transthoracic needle biopsy. He had normal pulmonary function tests. His CT chest showed a $10.1 \mathrm{~cm} \times 9.0 \mathrm{~cm} \times 8.0 \mathrm{~cm}$ heterogenous anterior $\mathrm{MM}$ with calcification (Figure 1). There was also associated an elevated right hemidiaphragm suggesting phrenic nerve involvement (Figure 2). The tumor marker panel was within normal limits. The biopsy specimen confirmed this to be a thymoma.

He underwent an uncomplicated right hemi clamshell incision and radical thymectomy with en-bloc right phrenic nerve and pericardial resection. He did well post-operatively and was discharged on post-operative day 2. His final pathology returned as a type B1 thymoma with negative margins, T3N0M0 (Figure 2). He was evaluated in clinic 2 weeks later and was found to be functioning well at home (Figure 3). However, he did note new onset and increasing dyspnea and orthopnea. He was reevaluated the following week and his respiratory status had declined further. $\mathrm{He}$ now had associated ptosis, dysphagia and fatigue. He was admitted to hospital for evaluation and treatment. CT pulmonary angiography did not show pulmonary emboli. A moderate sized left pleural effusion was drained via thoracentesis. On assessment by a neurologist, he showed clinical signs and symptoms consistent with $M G$ and as such he was promptly started on pyridostigmine $(60 \mathrm{mg}$ four times daily), prednisone (60 $\mathrm{mg}$ daily) and IVIG ( $2 \mathrm{~g} /$ day for 2 days). His symptoms stabilized, however, approximately 4 days later, he sustained a witnessed apneic arrest resulting in pulseless electrical activity. He underwent chest compressions and return of spontaneous circulation occurred after 2 minutes. He was intubated and transferred to the intensive care unit with initiation of therapeutic hypothermia for 24 hours. He underwent further CT of his chest, abdomen and head which did not reveal any significant acute abnormalities to explain the apneic arrest. Upon rewarming, the patient did not show signs of neurological insult and was extubated 48 hours after. $\mathrm{He}$ tolerated extubation well, supported with bilevel positive airway pressure (BiPAP) ventilation. An AChR antibody assay was elevated confirming the diagnosis of MG. The remainder of his 30-day hospital course was dedicated towards improved physical conditioning, respiratory support and control of his MG symptoms. He was discharged on high doses of prednisone ( $60 \mathrm{mg}$ once daily), pyridostigmine 

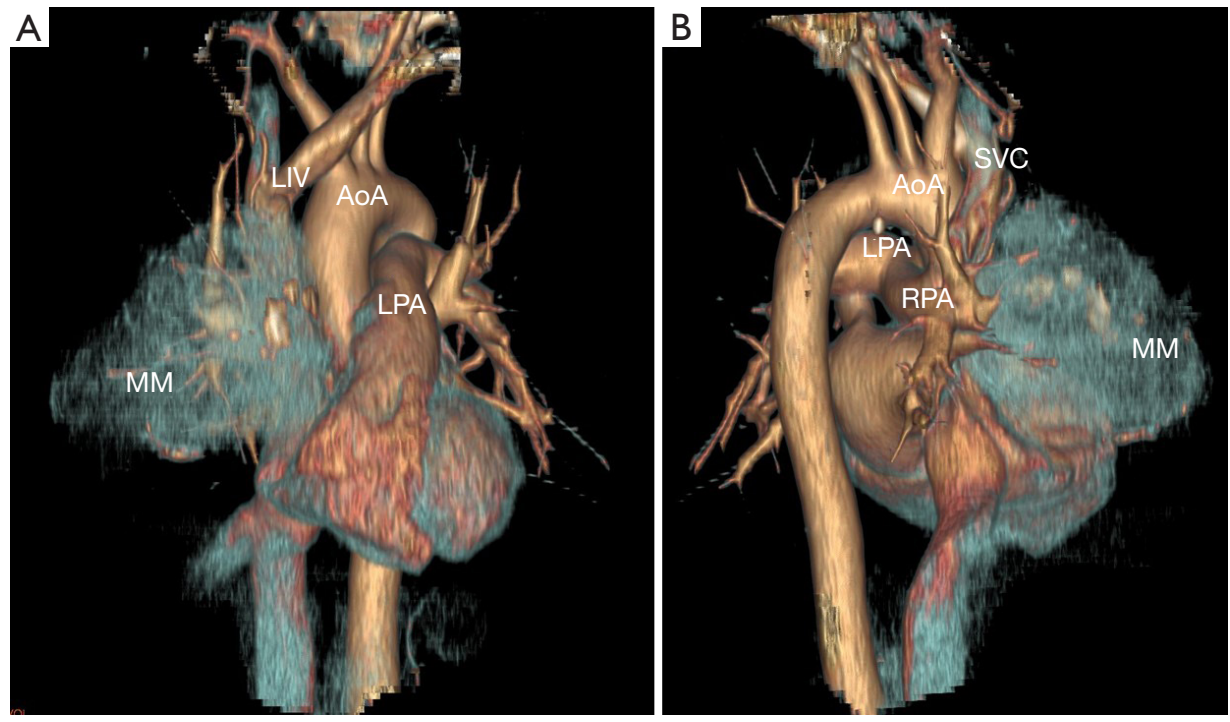

Figure 1 A three-dimensional reconstruction of the MM highlighted in light blue. (A) Left-sided view of the MM; (B) right-sided view of the MM. AoA, aortic arch; LPA, left pulmonary artery; RPA, right pulmonary artery; SVC, superior vena cava; MM, mediastinal mass.

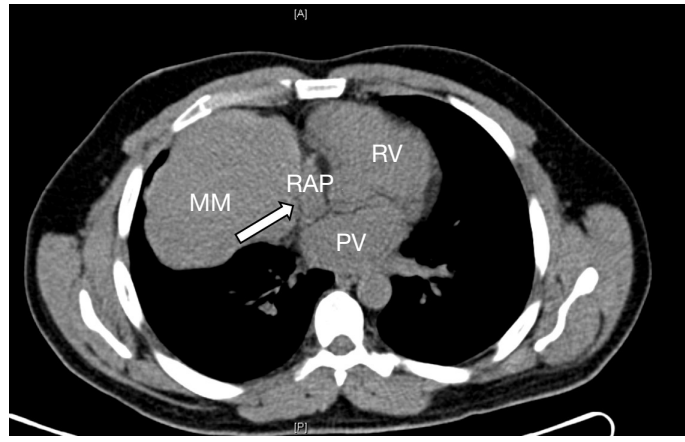

Figure 2 Large anterior MM with involvement of the phrenic nerve (arrow points to expected location of phrenic nerve). MM, mediastinal mass; RAP, right atrial appendage; PV, pulmonary veins; $\mathrm{RV}$, right ventricle.

(60 $\mathrm{mg}$ once to four timed a day as needed) and nocturnal BiPAP. He was weaned off prednisone and transitioned to azathioprine $(1 \mathrm{mg} / \mathrm{kg} /$ day $)$ as an outpatient over the course of 8 weeks without any evidence of a MG flair.

He had routine follow-up with his neurologist and was found to be in remission. At the 6 months mark he was weaned off azathioprine and tolerated this well. He was evaluated annually with a contrast enhanced CT chest as well as history and physical with a thoracic surgeon for his thymoma. He is currently 5 years post resection with no evidence of recurrence of thymoma. He is not on any medications for his MG. He continues to use nocturnal BiPAP likely secondary to the elevated right hemidiaphragm. He has otherwise returned to the same level of functional activity as he tolerated pre-operatively. All procedures performed in this study were in accordance with the ethical standards of the institutional and national research committees and with the Helsinki Declaration (as revised in 2013). Written informed consent was obtained from the patient for publication of this case report. A copy of the written consent is available for review by the editorial office of this journal.

\section{Discussion}

Patients with thymoma may develop several associated paraneoplastic syndromes, with MG the most frequent. It has been estimated that MG develops in approximately $25 \%$ of patients with thymoma. It is also estimated that approximately $20 \%$ of patients diagnosed with MG present initially in crisis. Patients who present in crisis under the age of 55 are 4 times more likely to be female. Patients with certain subtypes of thymoma are more likely to develop MG compared to other. Patients with subtypes $A$ and $A B$ possess MG $15-20 \%$ of the time, while patients with subtypes B1-B3 possess MG $40-50 \%$ of the time. Patients with more advanced Masaoka stage at diagnosis have a lower likelihood of possessing MG (7). This ranges from 19-67\% in Masaoka stage I to $1-15 \%$ in Masaoka stage IV. Patients with a T3N0M0 (Masaoko stage III) type B1 thymoma, like 


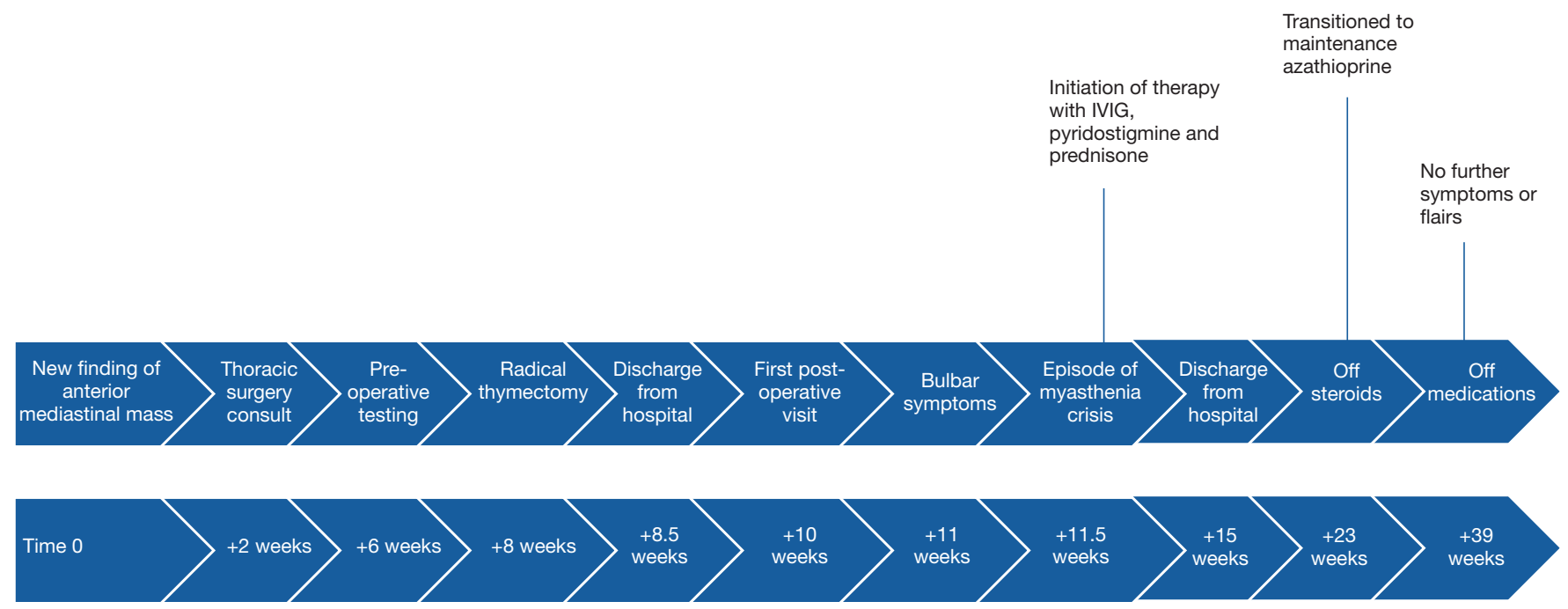

Figure 3 Timeline from initial discovery of thymoma to discharge post myasthenic crisis. IVIG, intravenous immunoglobulin.

this patient, have been found to have MG approximately $9 \%$ of the time (8).

Other risk factors for the development of MG in patients with thymoma include (I) detection of AChR autoantibodies, (II) development of B1 or B2 thymoma subtypes, (III) presence of ectopic thymic germinal centers, (IV) local invasiveness of thymoma, and (V) females under 50 years old (9). The patient in this case had two of the risk factors listed. However, it was only after onset of symptoms that the assay for AChR autoantibodies was sent. Detection of AChR autoantibodies pre-operatively would not have changed management in an asymptomatic patient. However, it may have raised the index of suspicion for onset of MG and lower the threshold for starting therapy on the patient's first post-operative visit in which he displayed mild bulbar symptoms. It would have also prompted a pre-operative evaluation by a neurologist who would remain involved in the care of this patient should symptoms have arisen. As such, this case suggests that all patients who are referred for resection for thymoma should be tested for AChR autoantibodies. A pre-operative neurologist consultation should be performed in those patients testing positive, for a complete evaluation and recommendations regarding perioperative care. There are multiple guidelines which provide recommendations regarding the evaluation of patients with thymoma. However, only the European Society of Medical Oncologists (ESMO) makes any recommendation regarding the routine use of AChR testing in asymptomatic patients (10). The National Cancer Care Network (NCCN) does recommend evaluation for MG in all patients with thymoma. Nevertheless, the recommendation only suggests looking for signs and symptoms for MG and no specific biochemical or neurological testing (11).

There are several known triggers of MG. This list includes medications (fluoroquinolone and macrolide class of antibiotics, beta blockers and various antipsychotic class medications), surgery, pain as well as emotional and environmental stressors (12). The patient in this case could have been triggered by some of the variables listed above. As such, a myasthenia flair would have been expected in the early post-operative period instead of 3 weeks after thymectomy. At the 3-week mark, the surgical insult has been well established and its impact dissipating. Consequently, pain should play a less prominent role in triggering MG. The patient also did not receive any postoperative antibiotics or beta blockers. It remains unclear why this patient developed MG in a delayed fashion. This case highlights the need for a more sensitive mechanism to detect MG in patients with thymoma.

This case demonstrates an uncommon presentation of MG in an expected low risk profile thymoma patient. The availability of pre-operative subjective (signs/symptoms) and objective evidence (pulmonary function test findings) followed by AChR positive testing post-operatively well illustrates why current guidelines do not provide recommendations sensitive enough to detect $M G$ in patients with thymoma. The surgical approach and extent of resection is not representative of most thymomas that 
surgeons would encounter. Accordingly, this patient had an elevated hemidiaphragm that may have confounded the post-operative course. This patient may have experienced minor dyspnea in the post-operative period which would have been attributed to impaired chest wall mechanics secondary to the hemi clamshell incision and the phrenic nerve resection. As such, this represents a confounder that may have masked a flair occurring after surgery. However, his ability to ambulate well enough to be discharged and present two and half weeks later goes against this. For the myasthenic crisis this patient was treated with IVIG and maintained on azathioprine. This practice may not be commonplace in many parts of the world depending on availability of donor blood and drug formularies which limits the generalizability of our management of a myasthenic crisis.

\section{Conclusions}

The symptom burden of a patient pre-operatively does not always predict an indolent post-operative course. Moreover, even asymptomatic patients with no known diagnosis of MG may develop this disease in the post-operative period. Overall, this patient's demographic, final tumor pathology, uncomplicated post-operative stay and time course of symptoms would suggest that this patient should have a low likelihood of having MG, let alone develop a crisis. As such, this case highlights the importance of being vigilant in monitoring for MG regardless of patient variables and time lapsed after surgery. The use of routine pre-operative AChR testing may help identify patients who would benefit from earlier neurology consultation and involvement.

\section{Acknowledgments}

Funding: None.

\section{Footnote}

Reporting Checklist: The authors have completed the CARE reporting checklist. Available at https://ccts.amegroups. com/article/view/10.21037/ccts-2020-06/rc

Conflicts of Interest: All authors have completed the ICMJE uniform disclosure form (available at https://ccts. amegroups.com/article/view/10.21037/ccts-2020-06/coif). The authors have no conflicts of interest to declare.
Ethical Statement: The authors are accountable for all aspects of the work in ensuring that questions related to the accuracy or integrity of any part of the work are appropriately investigated and resolved. All procedures performed in this study were in accordance with the ethical standards of the institutional and national research committees and with the Helsinki Declaration (as revised in 2013). Written informed consent was obtained from the patient for publication of this case report. A copy of the written consent is available for review by the editorial office of this journal.

Open Access Statement: This is an Open Access article distributed in accordance with the Creative Commons Attribution-NonCommercial-NoDerivs 4.0 International License (CC BY-NC-ND 4.0), which permits the noncommercial replication and distribution of the article with the strict proviso that no changes or edits are made and the original work is properly cited (including links to both the formal publication through the relevant DOI and the license). See: https://creativecommons.org/licenses/by-nc-nd/4.0/.

\section{References}

1. Gomez AM, Van Den Broeck J, Vrolix K, et al. Antibody effector mechanisms in myasthenia gravis-pathogenesis at the neuromuscular junction. Autoimmunity 2010;43:353-70.

2. Zisimopoulou P, Brenner T, Trakas N, et al. Serological diagnostics in myasthenia gravis based on novel assays and recently identified antigens. Autoimmun Rev 2013;12:924-30.

3. Farmakidis C, Pasnoor M, Dimachkie MM, et al. Treatment of myasthenia gravis. Neurol Clin 2018;36:311-37.

4. Thanvi BR, Lo TC. Update on myasthenia gravis. Postgrad Med J 2004;80:690-700.

5. Jaretzki A 3rd, Barohn RJ, Ernstoff RM, et al. Myasthenia gravis: recommendations for clinical research standards. Task Force of the Medical Scientific Advisory Board of the Myasthenia Gravis Foundation of America. Neurology 2000;55:16-23.

6. Wolfe GI, Kaminski HJ, Aban IB, et al. Randomized trial of thymectomy in myasthenia gravis. $\mathrm{N}$ Engl J Med 2016;375:511-22.

7. Ruffini E, Filosso PL, Mossetti C, et al. Thymoma: inter-relationships among World Health Organization 
histology, Masaoka staging and myasthenia gravis and their independent prognostic significance: a single-centre experience. Eur J Cardiothorac Surg 2011;40:146-53.

8. Weis CA, Yao X, Deng Y, et al. The impact of thymoma histotype on prognosis in a worldwide database. J Thorac Oncol 2015;10:367-72.

9. Lefeuvre CM, Payet CA, Fayet OM, et al. Risk factors associated with myasthenia gravis in thymoma patients: The potential role of thymic germinal centers. J Autoimmun 2020;106:102337.

10. Girard N, Ruffini E, Marx A, et al. Thymic epithelial

doi: $10.21037 /$ ccts-2020-06

Cite this article as: Nayak R, Rebello R, Shargall Y. New diagnosis of myasthenia gravis presenting as myasthenic crisis after radical thymectomy: a case report. Curr Chall Thorac Surg 2021;3:20. tumours: ESMO Clinical Practice Guidelines for diagnosis, treatment and follow-up. Ann Oncol 2015;26 Suppl 5:v40-55.

11. National Cancer Care Network Guidelines. Thymoma and Thymic Carcinoma. Version 1.2020 (Nov 27, 2019). Available online: https://www.nccn.org/professionals/ physician_gls/pdf/thymic_blocks.pdf (Access Date: June 18, 2020).

12. Wendell LC, Levine JM. Myasthenic crisis. Neurohospitalist 2011;1:16-22. 